\title{
Polarized excitons in nanorings and the 'optical' Aharonov-Bohm effect
}

\author{
A. O. Govorov, ${ }^{1,2}$ S. E. Ulloa, ${ }^{1}$ K. Karrai,${ }^{3}$ and R. J. Warburton ${ }^{4}$ \\ ${ }^{1}$ Department of Physics and Astronomy, and Nanoscale and Quantum \\ Phenomena Institute, Ohio University, Athens, Ohio 45701-2979 \\ ${ }^{2}$ Institute of Semiconductor Physics, Russian Academy of Sciences, Siberian Branch, 630090 Novosibirsk, Russia \\ ${ }^{3}$ Center for NanoScience, LMU, Geschwister-Scholl-Platz 1, 80539 München, Germany \\ ${ }^{4}$ Department of Physics, Heriot-Watt University, Edinburgh, UK
}

(Dated: October 30, 2018)

\begin{abstract}
The quantum nature of matter lies in the wave function phases that accumulate while particles move along their trajectories. A prominent example is the Aharonov-Bohm phase, which has been studied in connection with the conductance of nanostructures. However, optical response in solids is determined by neutral excitations, for which no sensitivity to magnetic flux would be expected. We propose a new mechanism for the topological phase of a neutral particle, a polarized exciton confined to a semiconductor quantum ring. We predict that this magnetic-field induced phase may strongly affect excitons in a system with cylindrical symmetry, resulting in switching between 'bright' exciton ground states and novel 'dark' states with nearly infinite lifetimes. Since excitons determine the optical response of semiconductors, the predicted phase can be used to tailor photon emission from quantum nanostructures.
\end{abstract}

PACS numbers: 78.67.Hc, 73.21.La, 73.23.Ra

It is known that the quantum mechanical phase of a state wave function is not a physical observable. This understanding, true in its absolute form, does not preclude the important possibility of observing relative phases in a suitably prepared system. In fact, much of the physics in meso- and nanoscopic systems is intrinsically connected to interference or phase-shift phenomena that manifest themselves in a number of experimentally measurable quantities. Prominent examples include the superconducting quantum interference devices (SQUIDs) [1] the quantum-phase factors induced by adiabatic changes (known as geometric Berry phases) [2], their generalization to non-adiabatic changes due to Aharonov and Anandan [3], and the well known Aharonov-Bohm (AB) effect [- 1 . Such a case appears naturally in systems with ring geometry in the presence of a magnetic field. In fact, recently available semiconductor rings $\simeq 10-100 \mathrm{~nm}$ in diameter allow one to explore this interesting physics in readily attainable magnetic fields. We report here on a new mechanism of phase difference acquired in a magnetic field by a composite and polarizable object with overall zero charge. Such neutral particles, excitons, are bound states of an electron and a hole in semiconductors, and are responsible for optical emission of crystals at low temperatures. The predicted interference effect has important observable consequences, as it affects the exciton emission and lifetime in nanoscopic semiconducting rings, and provides a novel phase interference phenomenon, the 'optical' AB effect. In particular, we predict a striking effect: the exciton emission can be strongly suppressed in certain magnetic-field windows.

The AB phase is most simply described as the phase acquired by a charge as it traverses a region where a magnetic flux exists, while no effects of the classical Lorentz force are present. The $\mathrm{AB}$ effect has been verified in a number of beautiful experiments using superconducting rings [5], where electrons move in the regions with zero magnetic field. In semiconductors, the $\mathrm{AB}$ effect has been used in fascinating experiments to measure the relative phases of alternative paths in electronic interferometers [6], and proposed as a mechanism to distinguish singlet and triplet states in coupled quantum dots, in connection with their use in solid state quantum computers [7, 8].

The exciton bound state of electron and a corresponding hole is a neutral object basically insensitive to external magnetic fields or fluxes (except for a weak diamagnetic behavior). In fact, excitons in quantum dots a few tens of nanometers in radius and an even smaller height, have been studied in recent times 19, 10, 11, 12. Moreover, thanks to the infrared absorption studies of Lorke et al., we know that semiconductor quantum rings are possible [13, where the electrons are coherent around the ring and non-localized. In this type of structures excitons in individual nanorings in strong magnetic fields can be generated and monitored using near-field scanning optics techniques [9, 14. Indeed, micro-photoluminescence $(\mu \mathrm{PL})$ experiments in individual quantum rings would provide an opportunity to explore the $\mathrm{AB}$ effect in neutral excitons 115, 16. We should emphasize that the $\mathrm{AB}$ oscillations of the ground state are predicted to be negligible at typical size/fields in the $1 \mathrm{D}$ rings 16. However, as we explain below, the in-plane radial polarization of the exciton in finite-width quantum rings produces a strong modulation of the oscillator strength of the excitonic transition with magnetic field, due to the $\mathrm{AB}$ effect.

Figure 1a illustrates Berry's gedanken experiment to explain how the adiabatic evolution of the Hamiltonian in a system gives rise to an additional phase [2]. The Berry phase is accumulated as the particle placed in an isolating box is slowly rotated around the field lines. Since 
the evolution is adiabatic, the system returns to its original state after a full cycle, and only a topological phase accumulates. The phase is given by the total flux in terms of the flux quantum, $\Phi / \Phi_{0}$, and is equivalent to the $\mathrm{AB}$ phase. When the object is a composite of two particles, one can imagine a similar adiabatic evolution, although here we rotate a polarized exciton having a radial dipole moment. After one full revolution, the particles obtain topological phases again, but these phases will be different, since the electron and hole have different trajectories. The phase difference is due to the unequal magnetic fluxes penetrating their paths. It is important, however, that the net relative phase would be effectively sampled/measured when the two carriers in the system combine to emit the photon seen in the PL experiment. The accumulated phase shift depends intrinsically on the polarizability of the composite object, and can even make optical transitions impossible, resulting in suppression of optical emission in well-defined windows of magnetic field. We demonstrate this effect using a model of a polarized exciton rotating in a quantum ring $(\mathrm{QR})$. Notice that topological phases on point dipoles have been considered [17], but their internal structure was not of interest in that case.

Today's semiconductor technology offers a variety of nanometer-size structures, including self-assembled QR's [13, 18], and type-II quantum dots [19, 20. In a ringshaped nanostructure, any asymmetry in the confinement potential may cause the polarization of excitons in the radial direction (Fig. 1c), so that an electron and hole may move along different circles. In fact, the existence of such an asymmetry is natural since an electron and hole have very different masses and move in different potentials, $U_{e}$ and $U_{h}$ [21]. Namely, in selfassembled QR's (Fig. 1c), the lighter electron tends to tunnel toward the ring center, whereas the heavier hole is localized mostly near the ring radius, producing a net radial polarization [20. Such polarization is easily calculated from a parameterized model of a quantum ring, $U_{e(h)}=m_{e(h)} \Omega_{e(h)}^{2}\left(r_{e(h)}-R_{r}\right)^{2} / 2$, where $r$ is the distance to the center of the ring, and $m_{e(h)}$ and $\Omega_{e(h)}$ are the effective masses and single-particle confinement frequencies, respectively. Moreover, the radial polarization in the exciton can appear also due to the deformation potential in a QR, which can produce drastically different effective potentials in the valence and conduction bands. The structural in-plane radius of a QR determined from atomic force micrographs is about 40nm (Fig. 1c), however, the electronic radius $R_{r}$ estimated from optical experiments turns out to be much smaller, $R_{r} \simeq 16 \mathrm{~nm}[13$. As the vertical, $z$-extension of the QR's is much smaller than their lateral size, we consider here only the in-plane motion, assuming strong quantization in the $z$-direction. In the case of type-II quantum dots, the radial dipole moment in an exciton appears because the quantum dot structure potential in its center forms a barrier for elec- trons and a well for holes. This situation results then in the electron and hole moving along different circles [22]. To describe excitons in both of these systems, we use a model of two 'parallel' one-dimensional rings, one for the electron and another for the hole (insert in Fig. 2), having different radii, $R_{e}$ and $R_{h}$, respectively.

The Hamiltonian of the exciton in this model reads

$$
\hat{H}_{t o t}=\hat{T}_{e}+\hat{T}_{h}+U_{e}+U_{h}+U_{C}\left(\left|\mathbf{r}_{e}-\mathbf{r}_{h}\right|\right),
$$

where $\mathbf{r}_{e(h)}$ are the in-plane coordinates, $\hat{T}_{e(h)}$ are the kinetic energies in the presence of a normal magnetic field, and $U_{C}$ is the Coulomb potential. Now we assume that the quantization in the radial direction is stronger than that in the azimuthal direction. It allows us to separate variables in the exciton wave function, $\Psi\left(\mathbf{r}_{e}, \mathbf{r}_{h}\right)=f_{e}\left(\rho_{e}\right) f_{h}\left(\rho_{h}\right) \psi\left(\theta_{e}, \theta_{h}\right)$. Here $\mathbf{r}=(\rho, \theta)$. The radial wave functions $f_{e(h)}$ are strongly localized near the radii, $R_{e(h)}$. The Hamiltonian describing the angular wave function $\psi\left(\theta_{e}, \theta_{h}\right)$ is (up to a $B$-independent constant term),

$$
\begin{aligned}
\hat{H}_{e x c} & =-\frac{\hbar^{2}}{2 m_{e} R_{e}^{2}} \frac{\partial^{2}}{\partial \theta_{e}^{2}}-\frac{i \hbar \omega_{e}}{2} \frac{\partial}{\partial \theta_{e}}-\frac{\hbar^{2}}{2 m_{h} R_{h}^{2}} \frac{\partial^{2}}{\partial \theta_{h}^{2}} \\
& +\frac{i \hbar \omega_{h}}{2} \frac{\partial}{\partial \theta_{h}}+\frac{m_{e} \omega_{e}^{2} R_{e}^{2}+m_{h} \omega_{h}^{2} R_{h}^{2}}{8} \\
& +u_{C}\left(\left|\theta_{e}-\theta_{h}\right|\right),
\end{aligned}
$$

where $\omega_{e(h)}=|e| B /\left[m_{e(h)} c\right]$ are the cyclotron frequencies of the particles, $B$ is the normal magnetic field, and $u_{c}$ is the Coulomb potential averaged over the coordinate $\rho$ involving the radial wave functions. Now it is convenient to introduce new variables, $\Delta \theta=\theta_{e}-\theta_{h}$, and $\theta_{0}=\left(a \theta_{e}+b \theta_{h}\right) /(a+b)$, where $\theta_{e(h)}$ is the angular coordinates of each particle, $a=m_{e} R_{e}^{2}$, and $b=m_{h} R_{h}^{2}$. The Hamiltonian then reads $\hat{H}_{\text {exc }}=\hat{H}_{\text {rot }}\left(\theta_{0}\right)+\hat{H}_{\text {int }}(\Delta \theta)$, where the translation of the whole exciton around the ring and the internal motion, are separate. The eigenfunctions are of the form $\psi\left(\theta_{e}, \theta_{h}\right)=\psi_{0}\left(\theta_{0}\right) \psi_{1}(\Delta \theta)$. Here, $\hat{H}_{\text {rot }}$ is given by

$$
\hat{H}_{\text {rot }}\left(\theta_{0}\right)=\varepsilon_{0}\left[-i \frac{\partial}{\partial \theta_{0}}+\frac{\Phi_{\Delta R}}{\Phi_{0}}\right]^{2},
$$

where $\varepsilon_{0}=\hbar^{2} /\left(2 R_{0}^{2} M\right), R_{0}=\left(R_{e}+R_{h}\right) / 2, M=$ $\left(m_{e} R_{e}^{2}+m_{h} R_{e}^{2}\right) / R_{0}^{2}$, and $\Phi_{\Delta R}=\pi\left(R_{e}^{2}-R_{h}^{2}\right) B=$ $2 \pi \Delta R R_{0} B$ is the magnetic flux penetrating the area between the electron and hole trajectories (Fig. 1b); and $\Delta R=R_{e}-R_{h}$.

The internal-motion Hamiltonian $\hat{H}_{\text {int }}$ involves the Coulomb potential. In small QR's, the quantization due to kinetic motion is much stronger than the Coulomb interaction, and the picture is basically that of single particles. This limit corresponds to $R_{e(h)}<a_{0}^{*}$, where $a_{0}^{*}$ is the effective Bohr radius in the semiconductor. In large QR's, where $R_{e(h)}>a_{0}^{*}$, the motion becomes strongly 
correlated, as the particles form a tightly-bound exciton and move together around the ring.

It is possible to obtain analytic solutions for the spectrum in these two limiting cases. For small QR's, the electron and hole move independently (Fig. 2, insert). There, the exciton spectrum is given by

$E_{e x c}=E_{g}+\frac{\hbar^{2}}{2 m_{e} R_{e}^{2}}\left(L_{e}+\frac{\Phi_{e}}{\Phi_{0}}\right)^{2}+\frac{\hbar^{2}}{2 m_{h} R_{h}^{2}}\left(L_{h}-\frac{\Phi_{h}}{\Phi_{0}}\right)^{2}$,

where $E_{g}$ is the magnetic field-independent term which includes the band-gap energy. $L_{e}$ and $L_{h}$ represent the angular momenta. The magnetic field $B$ enters Eq. (4) through the magnetic fluxes $\Phi_{e(h)}=\pi R_{e(h)}^{2} B$, which describe the quantum phase accumulating in the wave function of each particle as it travels along the ring.

For the limit of the tightly-bound exciton (Fig. 3, insert), the spectrum is determined by $\hat{H}_{\text {rot }}$ and takes the form

$$
E_{e x c}^{\prime}=E_{g}^{\prime}+\frac{\hbar^{2}}{2 M R_{0}^{2}}\left(L+\frac{\Delta \Phi}{\Phi_{0}}\right)^{2},
$$

where $L=L_{e}+L_{h}$ is the total momentum, $M, R_{0}$ are as above, and $E_{g}^{\prime}$ is field-independent. The net magnetic flux through the area between the electron and hole trajectories, $\Delta \Phi$, is proportional to the radial dipole moment of the exciton, $D=-e\left(R_{e}-R_{h}\right)$.

The $B$-dependent terms from Eqs. (14) and (5) are shown in the upper panels of Figs. 2 and 3. Since emission spectra of semiconductors at low temperatures are governed by the exciton ground-state, one must pay special attention to the lowest energy states. The figures show that the ground state changes with increasing magnetic field, and may acquire non-zero angular momentum values. For small quantum rings (Fig. 2), the ground state $\left(L_{e}, L_{h}\right)=(0,0)$ changes successively in favor of the states $(0,+1),(-1,+1),(-1,+2)$, etc., as the field increases, producing a sequence of ground state total angular momentum values of $0,1,0,1$, etc. On the other hand, for the strongly-correlated exciton in large rings (Fig. 3), the ground-state momentum changes increasingly from $L=0$ to $L=+1,+2,+3 \ldots$, with magnetic field.

Only the excitons with zero total momentum can emit photons. This condition arises as the photon's angular momentum is exhausted by the atomic wave functions involved in the optical transition. The optical selection rule $L=0$ means that the emission intensity of a QR can be dramatically suppressed in $B$-intervals where the exciton ground state acquires a non-zero momentum. This suppression implies that the exciton lifetime becomes significantly longer, as its dipole-allowed decay is forbidden. Such behavior is seen in the lower panels of Figs. 2 and 3. Thus, we predict a striking manifestation of the $\mathrm{AB}$ effect in the emission spectra of QR's at low temperatures. Of course, the emission intensity as a function of the magnetic field strongly depends on Coulomb correlations in the exciton (compare Figs. 2 and 3). However, the appearance of the $B$-windows in the emission of QR's of various sizes has a common origin - the electric dipole moment in the radial direction. The dipole moment is therefore responsible for the magnetic interference effect while the exciton moves along the QR. Previous models of QR's with $R_{e}=R_{h}$ could not (and did not) exhibit this important effect [15].

Our discussion implies the conservation of angular momentum in a QR which can be, in principle, destroyed by defects. However, as mentioned already, recent experiments on optical and electronic properties of QR's give strong evidence of coherent motion of electrons in these self-assembled nanostructures [13].

It is important to emphasize that these transitions depend on the net magnetic flux $\Delta \Phi$ through the area between the electron and hole trajectories (Fig. 1b). Due to the global conservation of angular momentum, the exciton spinning with nonzero momentum cannot emit a photon and becomes dark (very long lifetime) in welldefined intervals of magnetic field, which can be observed as noticeable 'blinking' PL response for different fields. This allows tailoring of excitonic transitions by switching the ground state from radiative to non-radiative wave function configurations.

The novel property of the topological Berry phase for a polarized exciton lies in the complex nature of this quasiparticle. For an electron and hole in the exciton, the difference of phases plays an important role rather than simply the phase of the single particle. Since the exciton 'stores' a photon, such a phase difference determines whether the QR is bright or dark in optical emission. This offers the unique opportunity to observe microscopic electronic phases in optical emission experiments.

We thank Axel Lorke for providing us with the quantum rings AF micrograph, and P. Petroff, J.P. Kotthaus, A.V. Chaplik, and A.V. Kalameitsev for useful discussions. We acknowledge support of US-DOE grant DEFG02-91ER45334, and the CMSS Program at OU.

[1] See, e.g., J.R. Kirtley, et al., Appl. Phys. Lett. 66, 1138 (1995).

[2] M.V. Berry, Proc. R. Soc. Lond. A392, 45 (1984). See also A. Shapere and F. Wilczek, eds., Geometric Phases in Physics (World Scientific, Singapore, 1989).

[3] Y. Aharonov and J. Anandan, Phys. Rev. Lett. 58, 1593 (1987).

[4] Y. Aharonov and D. Bohm, Phys. Rev. 115, 485 (1959).

[5] A. Tonomura, et al., Phys. Rev. Lett. 56, 792 (1986). M. Peshkin and A. Tonomura, The Aharonov-Bohm Effect (Springer Verlag, Berlin, 1989).

[6] E. Buks, et al., Nature 391, 871 (1998). 
[7] D. Loss and E.V. Sukhorukov, Phys. Rev. Lett. 84, 1035 (2000).

[8] A.W. Holleitner, C.R. Decker, H. Qin, K. Eberl, and R.H. Blick, Phys. Rev. Lett. 87, 256802 (2001).

[9] R.J. Warburton, et al., Nature 405, 926 (2000).

[10] M. Bayer, O. Stern, P. Hawrylak, S. Fafard, and A. Forchel, Nature 405, 923 (2000).

[11] F. Findeis, et al., Appl. Phys. Lett. 78, 2958 (2001).

[12] D. Mowbray, Phys. World 13, 27 (2000); P. Michler, et al., Science 290, 2282 (2000).

[13] A. Lorke, et al., Phys. Rev. Lett. 84, 2223 (2000).

[14] C. Obermuller, et al., Appl. Phys. Lett. 74, 3200 (1999).

[15] A.V. Chaplik, JETP Letters 62, 900 (1995); R.A. Römer and M.E. Raikh, Phys. Rev. B 62, 7045 (2000).

[16] J. Song and S.E. Ulloa, Phys. Rev. B 63, 125302 (2001); H. Hu, J.L. Zhu, D.J. Li, J.J. Xiong, Phys. Rev. B 63, 195307 (2001).

[17] J. Anandan, Phys. Rev. Lett. 85, 1354 (2000).

[18] J.M. Garcia, et al., Appl. Phys. Lett. 71, 2014 (1997).

[19] F. Hatami, et al., Appl. Phys. Lett. 67, 656 (1995); A.I.
Yakimov, et al., Phys. Rev. B 63, 045312 (2001); M. Hayne, et al., Phys. Rev. B 62, 10324 (2000).

[20] Although we have emphasized intrinsic potential asymmetries in self-assembled QR's (or type II dots), there are other alternatives. The QR can be fabricated by etching technology which allows the incorporation of top metallic gates. In such etched systems, a voltage applied to the metallic gate in the center of a $\mathrm{QR}$ may result in a tunable dipole moment for excitons.

[21] L. Jacak, P. Hawrylak, and A. Wojs, Quantum dots (Springer Verlag, Berlin, 1998).

[22] A.B. Kalameitsev, V.M. Kovalev, and A.O. Govorov, JETP Lett. 68, 669 (1998); K.L. Janssens, B. Partoens, and F.M. Peeters, Phys. Rev. B 64, 155324 (2001).

[23] In recent years, persistent currents in mesoscopic loops have triggered a lot of attention and activity. See e.g., C. Chapelier, D. Mailly, and A. Benoit, Festkorp.- Adv. Sol. State Phys. 34, 163 (1995). 

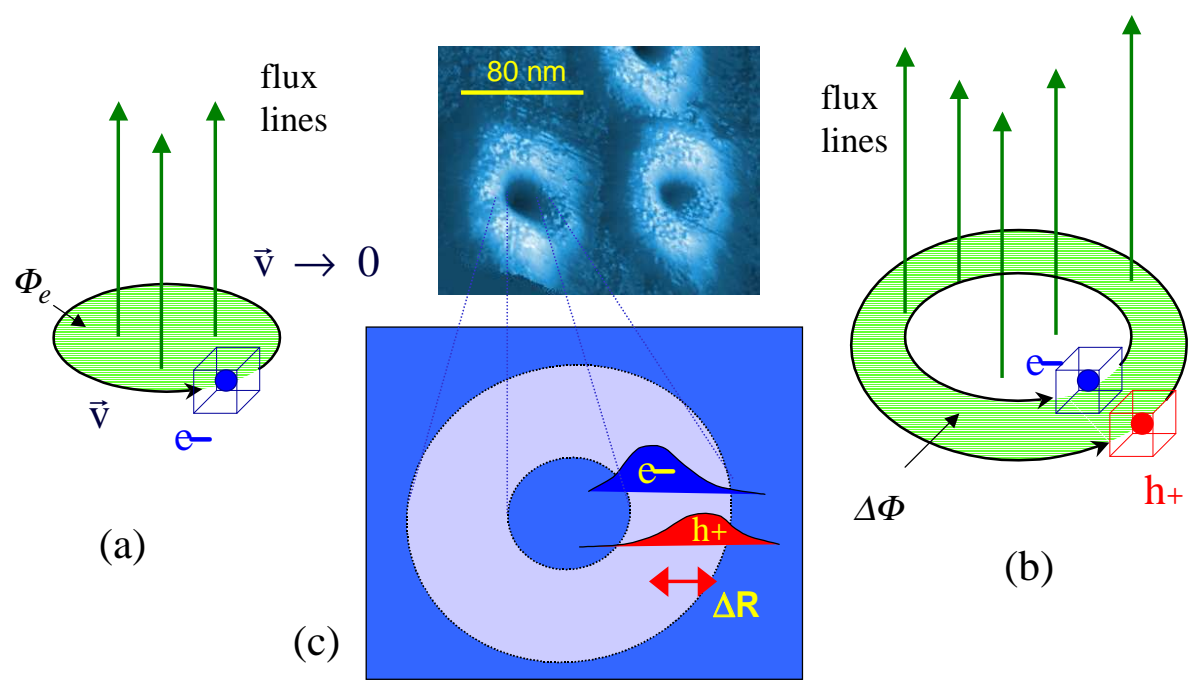

(b)

FIG. 1: (a) and (b) illustrate origin of Berry phase for a single charged particle and for an exciton, respectively. (a) An electron is placed in an isolating box and rotated along a closed path. The Berry phase, related in this case to the $\mathrm{AB}$ effect, is proportional to the magnetic flux penetrating the electron trajectory, $\Phi_{e}$. (b) For the polarized exciton, one can adopt the same picture, placing an electron and a hole in separate boxes. After a full revolution, the electron and hole accumulate different phases, yielding a relative phase in the pair proportional to the radial dipole moment. As the electron and hole induce opposite currents, the $\mathrm{AB}$ effect in this case originates from the magnetic flux through the area between the two trajectories, $\Delta \Phi$. (c) Atomic-force micrograph of self-assembled InAs nano-rings on a substrate of GaAs. Due to different effective masses, the rotating electron and hole are polarized in the radial direction. The electronic radius of nanorings is less that the structural one, according to spectroscopy experiments 13]. 


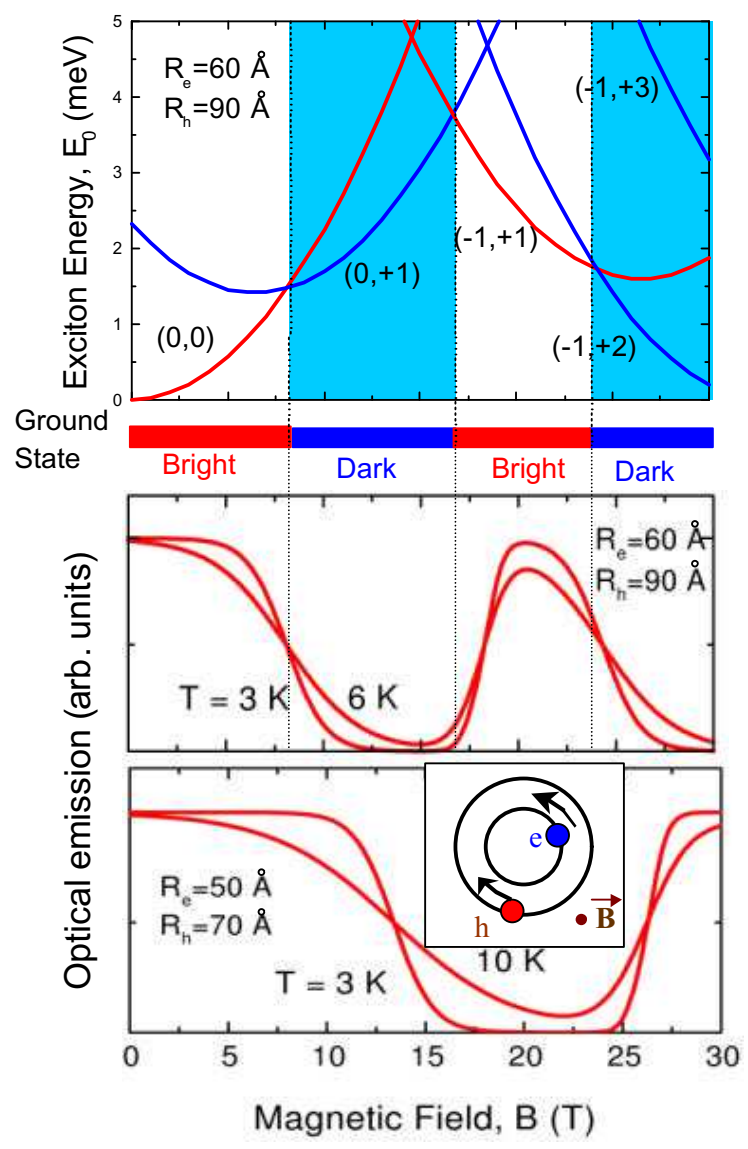

Fig. 2

FIG. 2: Exciton spectrum and emission intensity in two parallel rings for weak Coulomb interaction (small ring radii). Upper two panels are for $R_{e}=60 \AA$ and $R_{h}=90 \AA$; lower panel shows emission intensity for a smaller ring. Insert: uncorrelated motion of particles in a ring. At low temperature $T$ the exciton emission from a ring is strongly suppressed in well-defined windows of magnetic field which scale with ring radii. With increasing temperature the excited exciton states become accessible and the suppression is less pronounced, although strong oscillations with magnetic field remain. A "bright" ground state has $L=0$, and it is "dark" otherwise. 

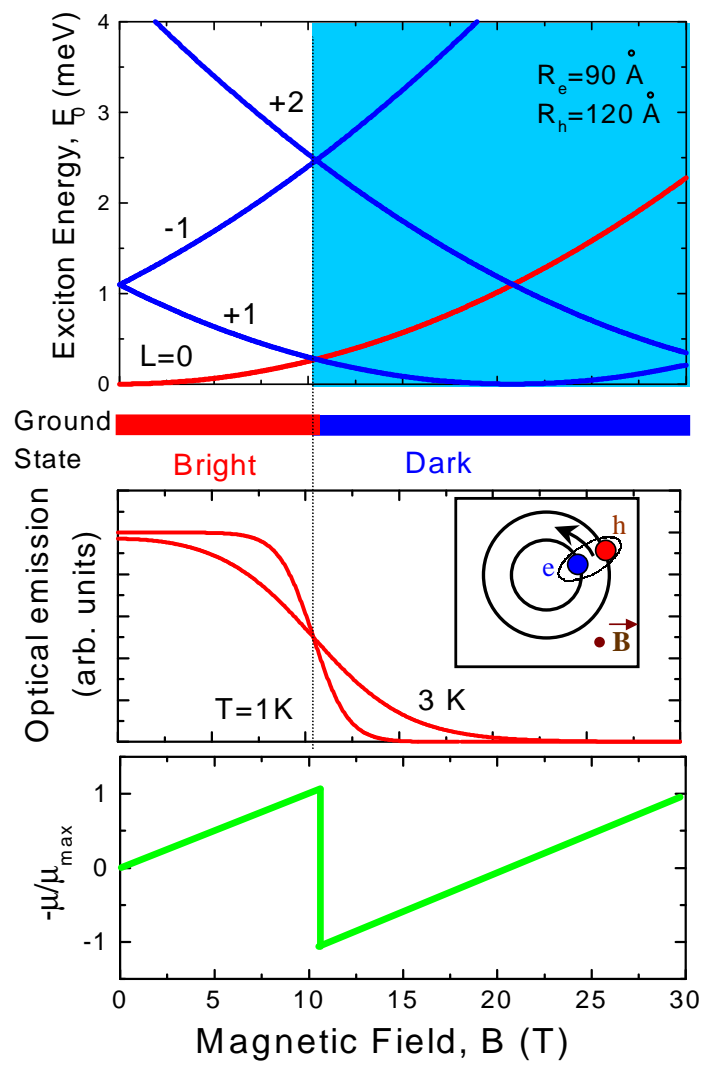

FIG. 3: Exciton spectrum, optical emission intensity, and magnetization in rings for strong Coulomb interaction (large ring radii). Insert: correlated motion of electron-hole pair in parallel rings. The character of exciton spectrum is different to the case of uncorrelated electron-hole pair (Fig. 2). This leads to the existence of a magnetic field threshold that is well expressed at low temperatures, $T$. Lowest graph illustrates magnetization of an exciton in the ground state at low temperature, $\mu=-\partial E_{e x c} / \partial B$, proportional to the persistent current in the ring [23]. Threshold for emission intensity coincides with the well-known jump in persistent current vs. flux. 